\title{
Dynamic Instability Analysis of a Rotating Ship Shaft under a Periodic Axial Force by Discrete Singular Convolution
}

\author{
Wei Li, ${ }^{1,2,3}$ Zhiwei Song, $^{1}$ Xuexia Gao, ${ }^{4}$ and Zhigang Chen ${ }^{5}$ \\ ${ }^{1}$ School of Naval Architecture and Ocean Engineering, Huazhong University of Science and Technology, Wuhan 430074, China \\ ${ }^{2}$ Hubei Key Laboratory of Naval Architecture \& Ocean Engineering Hydrodynamics (HUST), Wuhan 430074, China \\ ${ }^{3}$ Collaborative Innovation Center for Advanced Ship and Deep-Sea Exploration (CISSE), Shanghai 200240, China \\ ${ }^{4}$ Software and Technology College, Qingdao University, Qingdao 266101, China \\ ${ }^{5}$ China Ship Development and Design Center, Wuhan 430064, China
}

Correspondence should be addressed to Wei Li; hustliw@hust.edu.cn

Received 16 November 2014; Accepted 5 January 2015

Academic Editor: Tony Murmu

Copyright $\odot 2015$ Wei Li et al. This is an open access article distributed under the Creative Commons Attribution License, which permits unrestricted use, distribution, and reproduction in any medium, provided the original work is properly cited.

Dynamic instability of a rotating ship shaft subjected to a periodic axial force is studied by using discrete singular convolution (DSC) with regularized Shannon's delta kernel. The excitation frequency is related to the spinning speed and the number of blades on the propeller. Effects of number of blades, constant term in the periodic force, and damping on dynamic instability regions are investigated. The results have shown that the increase of number of blades and damping could improve the dynamic stability of rotating shaft with damping. The increase of constant term in the periodic force leads to dynamic instability regions shifting to lower frequencies, making the shaft more sensitive to periodic force. Those dynamic instability regions obtained by DSC method have been compared with those by Floquet's method to verify the application of DSC method to dynamic instability analysis of rotating ship shaft.

\section{Introduction}

When a ship sails at sea, the propulsion shaft is subjected to a periodic axial force induced by a rotating screw propeller. The excitation frequency of the periodic axial force has relation with spinning speed and the number of blades on the propeller. When the excitation frequency and the natural frequency of the shaft are in a certain condition, that may cause serious dynamic instability phenomenon [1]. In order to avoid this phenomenon happening and protect the safety of the rotating shaft, much attention should be paid to the dynamic instability analysis of such rotating ship shafts.

The dynamic instability analysis of rotating systems subjected to axial forces has been studied for many years. Chen and $\mathrm{Ku}[2,3]$ studied the dynamic stability of a rotating Timoshenko shaft and a rotating Timoshenko shaftdisk system, respectively. Lee $[4,5]$ analyzed the dynamic stability of spinning pretwisted cantilever beams subjected to axial pulsating loads and axial base excitations, respectively.
Then Lee et al. [6] studied dynamic stability of a spinning Timoshenko shaft with time-dependent spin rate by the use of the multiple scales method. And also Tan et al. [7] analyzed parametric instability of spinning pretwisted beams subjected to spin speed perturbation. Liao and Huang $[8,9]$ addressed parametric instability of a spinning pretwisted cantilever beam under periodic axial force and a cantilever pretwisted beam with time-dependent spinning rate, respectively. Chen and Peng [10] investigated the dynamic stability of a rotating composite shaft under axial periodic forces. Sheu and Chen [11] used a lumped mass model to study the parametric instability of a cantilever shaft-disk system subjected to axial and follower loads. Young and Gau [12, 13] studied the dynamic stability of a pretwisted cantilever beam with constant spin rates and nonconstant spin rates under axial random forces at the free end. Lin and Chen [14] presented dynamic stability problems of spinning pretwisted sandwich beams with a constrained damping layer subjected to periodic axial loads. Recently, Pei [15] analyzed the differences in 
the instability regions for gyroscopic system by Bolotin's method and Floquet's method. Pavlović et al. [16] studied the stochastic stability of an elastic balanced rotating shaft subjected to action of axial forces at the ends.

In these published works, the periodic axial forces are the type $P(t)=P_{S}+P_{D} \cos (\theta t)\left(P_{S}\right.$ is the constant part, $P_{D}$ is the amplitude of dynamic part with time $t$, and $\theta$ is the excitation frequency.) But, for the rotating ship shaft [17], the periodic axial forces made by rotating propeller can be expressed in a series; the type is $(t)=P_{S}+P_{D 1} \cos \left(Z_{p} \Omega t\right)+P_{D 2} \cos \left(2 Z_{p} \Omega t\right)+$ $P_{D 3} \cos \left(3 Z_{p} \Omega t\right)+\cdots$. The excitation frequencies of periodic axial forces are equal to the blade frequency $Z_{p} \Omega$ and its multiplies $\left(i Z_{p} \Omega, i=2,3, \ldots\right)$. The blade frequency is equal to the shaft spinning speed $\Omega$ multiplied by the number of blades $Z_{p} . P_{S}$ is the constant term; $P_{D 1}, P_{D 2}$, and $P_{D 3}$ are the amplitudes of dynamic terms. Compared to $P_{S}$ and $P_{D 1}$, the values of $P_{D 2}$ and $P_{D 3}$ are very small and could be neglected. So the axial force considered in this work is expressed as $P(t)=P_{S}+P_{D} \cos \left(Z_{p} \Omega t\right)$. Therefore, it is necessary to analyze the dynamic instability problem of a rotating ship shaft subjected to such type of periodic axial force. In this work, a relatively new method, discrete singular convolution (DSC) [18], is introduced for the solutions of dynamic responses and dynamic stability analysis of rotating shafts.

The discrete singular convolution method proposed by Wei [18] has emerged as a new technique for numerical solutions of differential equations. Compared to the other conventional numerical methods, the discrete singular convolution (DSC) has the global methods' high accuracy and the local methods' flexibility for handling complex geometry and boundary conditions in structural analysis. And this method is also able to accurately predict very high order vibration modes of plates and beams without numerical instability. Many studies have indicated that DSC has high accuracy and reliability for free vibration and buckling analysis of beams, plates, and shells. Wei and his cooperators applied the DSC method to vibration analysis of beams [19-22] and plates [23-29]. Civalek [30-33] gave numerical solutions of free vibration problems of truncated conical shells, laminated conical and cylindrical shells, and isotropic and orthotropic rectangular plates by using DSC method. To extend the research and application scope of the DSC method, Lim et al. [34] and Hou et al. [35] developed DSC-Ritz method for vibration analysis of thick plates and shallow shells. Xiang et al. [36] explored DSC-element method for vibration analysis of rectangular Mindlin plates. And then Xiang et al. [37] also explored DSC-Ritz element method for vibration analysis of rectangular Mindlin plates with mixed edge supports. These studies indicate that the DSC method works very well for the vibration analyses of beams, plates and shells, and so forth. Recently, Wang et al. [38] applied the DSC method to solve elastic wave propagation problem. Song et al. [39] used DSC method to analyze the dynamic stability and nonstationary vibration of beams.

The aim of the present paper is to propose a relatively new numerical approach using the method of DSC for the dynamic instability analysis of a rotating ship shaft under periodic axial force induced by propeller. The algorithm for solving equations of rotating shafts has been presented. The dynamic instability regions of rotating ship shaft are obtained through judging the dynamic stability of dynamic responses of transverse displacements obtained by using DSC method. Effects of number of blades, constant term in the periodic force, and damping on dynamic instability regions are discussed. And all these results by using DSC are compared with those obtained by Floquet's method to verify the application of DSC method to dynamic stability analysis of rotating shafts.

\section{Discrete Singular Convolution}

The method of discrete singular convolution (DSC) is an effective and useful approach for the numerical solutions of differential equations, which occur commonly in many science and engineering fields. The DSC method was originally introduced by Wei [18] as a simple and highly efficient numerical technique. This paper does not tell more details about this method. For more details of the mathematical background and application of the DSC method, interested readers may refer to the works of Wei et al. [19-23, 26,27]. The mathematical foundation of the DSC algorithm is the theory of distributions and wavelet analysis. For brevity, consider a distribution $T$ and $\eta(t)$ as an element of the space of the test function. A singular convolution can be defined by Wei [19]

$$
F(t)=(T * \eta)(t)=\int_{-\infty}^{\infty} T(t-x) \eta(x) d x,
$$

where $T(t-x)$ is a singular kernel. Depending on the form of the kernel $T$, the singular convolution is the central issue for a wide range of science and engineering problems, for example, singular kernels of delta type [20]

$$
T(x)=\delta^{(n)}(x) \quad(n=0,1,2, \ldots)
$$

where $\delta$ is the delta distribution and the superscript $n$ denotes the $n$ th-order distribution. Here, kernel $T(x)=\delta(x)$ is important for interpolation and $T(x)=\delta^{(n)}(x)(n=$ $1,2, \ldots)$ are essential for differentiations. However, since these kernels are singular, they cannot be directly digitized in computer. To avoid the difficulty of using singular expressions directly in computer, sequences of approximations $\left\{T_{\alpha}\right\}$ of the distribution $T$ can be constructed:

$$
\lim _{\alpha \rightarrow \alpha_{0}} \mathrm{~T}_{\alpha} \longrightarrow T(x)
$$

where $\alpha_{0}$ is a generalized limit. Obviously, in the case of $T(x)=\delta(x)$, the sequence $T_{\alpha}(x)$ is a delta sequence. Moreover, with a sufficiently smooth approximation, it is useful to consider a discrete singular convolution [19]

$$
F_{\alpha}(t)=\sum_{k} T_{\alpha}\left(t-x_{k}\right) f\left(x_{k}\right)
$$

where $F_{\alpha}(t)$ is an approximation to $F(t)$ and $\left\{x_{k}\right\}$ is an appropriate set of discrete points on which the DSC is well defined. Note that the original test function $\eta(x)$ has been replaced by $f(x)$. This new discrete expression is suitable for 


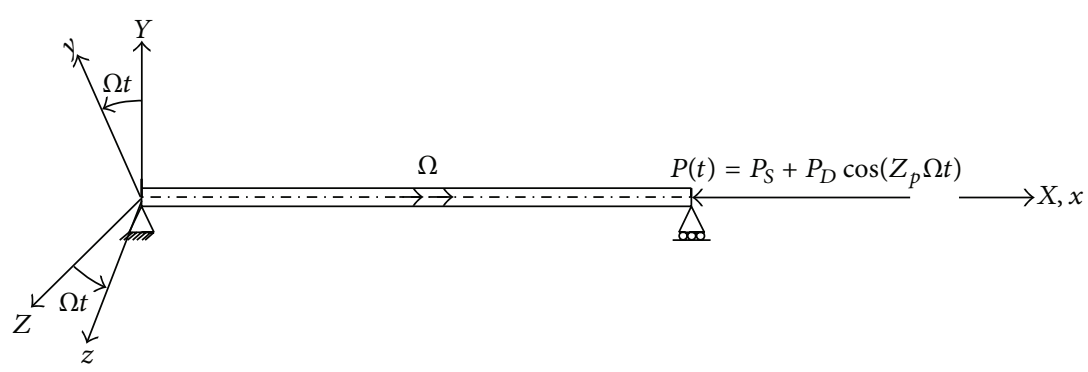

FIGURE 1: The rotating ship shaft and coordinate systems.

computer realization. Recently, the use of some new kernels and regularizer such as delta regularizer $[19,20,23,26]$ was proposed to solve applied mechanics problem. Shannon's kernel is regularized as [23]

$$
\begin{array}{r}
\delta_{\Delta, \sigma}\left(x-x_{k}\right)=\frac{\sin \left[(\pi / \Delta)\left(x-x_{k}\right)\right]}{(\pi / \Delta)\left(x-x_{k}\right)} \exp \left[-\frac{\left(x-x_{k}\right)^{2}}{2 \sigma^{2}}\right] \\
\sigma>0
\end{array}
$$

where $\Delta$ is the grid spacing. The parameter $\sigma$ determines the width of the Gaussian envelope and often varies in association with the grid spacing, $\sigma=r \Delta$. It is also known that the truncation error is very small due to the use of the Gaussian regularizer; the above formulation given by (5) is practically and has an essentially compact support for numerical interpolation. In the DSC method, the function $f(x)$ and its derivatives with respect to the $x$ coordinate at a grid point $x_{i}$ are approximated by a linear sum of discrete values $f\left(x_{k}\right)$ in a narrow bandwidth $\left[x-x_{M}, x+x_{M}\right]$. This can be expressed as [28]

$$
\begin{array}{r}
\left.\frac{d^{n} f(x)}{d x^{n}}\right|_{x=x_{i}}=f^{(n)}(x) \approx \sum_{k=-M}^{M} \delta_{\Delta, \sigma}^{(n)}\left(x_{i}-x_{k}\right) f\left(x_{k}\right) \\
(n=0,1,2, \ldots),
\end{array}
$$

where superscript $(n)$ denotes the $n$ th-order derivative with respect to $x$. When the regularized Shannon's delta (RSD) kernel is used, the detailed expressions for $\delta_{\Delta, \sigma}^{(n)}$ can be easily obtained. Readers can refer to some published references $[20,23]$.

\section{Theory and Algorithm}

3.1. Equation of Motion. Figure 1 illustrates a uniform simply supported Euler shaft rotating about its longitudinal axis with a constant spinning speed $\Omega$, subjected to a periodic axial force $P(t)=P_{S}+P_{D} \cos \left(Z_{p} \Omega t\right)$, and $(X Y Z)$ is the fixed coordinate system while $(x y z)$ is the coordinate system attached to the shaft with the $x$-axis aligned with the $x$ axis. Using Euler-Bernoulli beam theory, the effect of rotatory inertia is neglected. The equations $[9,12]$ of motion can be written as

$$
\begin{aligned}
& m \frac{\partial^{2} u_{y}}{\partial t^{2}}-2 m \Omega \frac{\partial u_{z}}{\partial t}-m \Omega^{2} u_{y}+C \frac{\partial u_{y}}{\partial t}+E I \frac{\partial^{4} u_{y}}{\partial x^{4}} \\
& \quad+P(t) \frac{\partial^{2} u_{y}}{\partial x^{2}}=0, \\
& m \frac{\partial^{2} u_{z}}{\partial t^{2}}+2 m \Omega \frac{\partial u_{y}}{\partial t}-m \Omega^{2} u_{z}+C \frac{\partial u_{z}}{\partial t}+E I \frac{\partial^{4} u_{z}}{\partial x^{4}} \\
& \quad+P(t) \frac{\partial^{2} u_{z}}{\partial x^{2}}=0
\end{aligned}
$$

where $u_{y}, u_{z}$ are the transverse displacements in the $y$-and $z$-direction, $m$ is the mass per unit length of shaft, and $C, E, I$, and $t$ are the viscous damping coefficient, Young's modulus, axial moment of inertia, and time, respectively. $P_{S}$ is the constant term, $P_{D}$ is the amplitude, and $Z_{p}$ is the number of the blades on the screw propeller.

For simplicity, introduce the following dimensionless quantities:

$$
\begin{gathered}
X=\frac{x}{l} \quad U_{Y}=\frac{u_{y}}{l} \quad U_{Z}=\frac{u_{z}}{l}, \\
\alpha=\frac{P_{S}}{P_{c r}} \quad \beta=\frac{P_{D}}{P_{c r}} \quad \bar{\Omega}=\frac{\Omega}{\omega}, \\
\zeta=\frac{C}{2 m \omega} \quad \tau=\omega t \quad \omega^{*}=\omega \sqrt{1-\alpha}, \\
\overline{\Omega^{*}}=\frac{\Omega}{\omega^{*}}=\frac{\bar{\Omega}}{\sqrt{1-\alpha}} \quad U=\sqrt{U_{Y}^{2}+U_{Z}^{2}},
\end{gathered}
$$

where $P_{c r}=\pi^{2} E I / l^{2}$ is the buckling force of a nonspinning simply supported shaft, $\omega=\pi^{2} \sqrt{E I / m l^{4}}$ is the natural bending frequency for the first mode, $\omega^{*}=\omega \sqrt{1-\alpha}$ is the natural bending frequency with constant axial compressive force, $l$ is the length of the shaft, $\zeta$ is damping ratio, $U$ is composition of $U_{Y}$ and $U_{Z}$, and $\bar{\Omega}$ is dimensionless spinning speed. 
Then, (7) can be written as

$$
\begin{gathered}
\frac{\partial^{2} U_{Y}}{\partial \tau^{2}}-2 \bar{\Omega} \frac{\partial U_{Z}}{\partial \tau}-\bar{\Omega}^{2} U_{Y}+2 \zeta \frac{\partial U_{Y}}{\partial \tau}+\frac{1}{\pi^{4}} \frac{\partial^{4} U_{Y}}{\partial X^{4}} \\
+\frac{\left(\alpha+\beta \cos \left(Z_{p} \bar{\Omega} \tau\right)\right)}{\pi^{2}} \frac{\partial^{2} U_{Y}}{\partial X^{2}}=0, \\
\frac{\partial^{2} U_{Z}}{\partial \tau^{2}}+2 \bar{\Omega} \frac{\partial U_{Y}}{\partial \tau}-\bar{\Omega}^{2} U_{Z}+2 \zeta \frac{\partial U_{Z}}{\partial \tau}+\frac{1}{\pi^{4}} \frac{\partial^{4} U_{Z}}{\partial X^{4}} \\
+\frac{\left(\alpha+\beta \cos \left(Z_{p} \bar{\Omega} \tau\right)\right)}{\pi^{2}} \frac{\partial^{2} U_{Z}}{\partial X^{2}}=0 .
\end{gathered}
$$

$$
\begin{gathered}
\frac{\partial^{2} U_{Z}}{\partial \tau^{2}}=-2 \bar{\Omega} \frac{\partial U_{Y}}{\partial \tau}+\bar{\Omega}^{2} U_{Z}-2 \zeta \frac{\partial U_{Z}}{\partial \tau}-\frac{1}{\pi^{4}} \frac{\partial^{4} U_{Z}}{\partial X^{4}} \\
-\frac{\left(\alpha+\beta \cos \left(Z_{p} \bar{\Omega} \tau\right)\right)}{\pi^{2}} \frac{\partial^{2} U_{Z}}{\partial X^{2}} .
\end{gathered}
$$

The boundary conditions are

$$
U(0, \tau)=U(1, \tau)=0, \quad \frac{\partial^{2} U(0, \tau)}{\partial X^{2}}=\frac{\partial^{2} U(1, \tau)}{\partial X^{2}}=0
$$

3.2. Discretization and Algorithm. The DSC discrete scheme (6) is utilized for the spatial discretization and the fourthorder Runge-Kutta (RK4) scheme is used for the time discretization $[39,40]$. The computational domain of coordinate $X$ is $[0,1]$, and the coordinate $X$ is equally spaced; the grid sizes are denoted by $\Delta X=(1-0) / N$ ( $N$ is the total number of partition grids on the computational domain $[0,1])$; the grid points are denoted by $X_{j}=(j-1) \Delta X(j=1,2, \ldots, N+1)$. So $X_{j}-X_{j+k}=-k \Delta X$. The approximate values of $U_{Y}$ and $U_{Z}$ at the grid point $X_{j}$ are expressed as $U_{Y, j}$ and $U_{Z, j}$ Then, (10) can be expressed as

$$
\begin{gathered}
\frac{\partial}{\partial \tau}\left[\begin{array}{c}
U_{Y, j} \\
U_{Z, j}
\end{array}\right]=\left[\begin{array}{c}
V_{Y, j} \\
V_{Z, j}
\end{array}\right], \\
\frac{\partial}{\partial \tau}\left[\begin{array}{c}
V_{Y, j} \\
V_{Z, j}
\end{array}\right] \\
=\left[\begin{array}{cc}
-2 \zeta & 2 \bar{\Omega} \\
-2 \bar{\Omega} & -2 \zeta
\end{array}\right]\left[\begin{array}{l}
V_{Y, j} \\
V_{Z, j}
\end{array}\right]+\left[\begin{array}{cc}
\bar{\Omega}^{2} & 0 \\
0 & \bar{\Omega}^{2}
\end{array}\right]\left[\begin{array}{l}
U_{Y, j} \\
U_{Z, j}
\end{array}\right] \\
-\left(\frac{1}{\pi^{4}} \frac{\partial^{4}}{\partial X^{4}}+\frac{\left(\alpha+\beta \cos \left(Z_{p} \bar{\Omega}^{2}\right)\right)}{\pi^{2}} \frac{\partial^{2}}{\partial X^{2}}\right)\left[\begin{array}{l}
U_{Y, j} \\
U_{Z, j}
\end{array}\right]
\end{gathered}
$$

Let

$$
\begin{aligned}
\left\{Y_{1, j}\right\} & =\left\{Y_{1,1}, Y_{1,2}, \ldots, Y_{1,2 N+2}\right\} \\
& =\left\{U_{Y, 1}, U_{Y, 2}, \ldots, U_{Y, N+1}, V_{Y, 1}, V_{Y, 2}, \ldots, V_{Y, N+1}\right\}, \\
\left\{Y_{2, j}\right\} & =\left\{Y_{2,1}, Y_{2,2}, \ldots, Y_{2,2 N+2}\right\} \\
& =\left\{U_{Z, 1}, U_{Z, 2}, \ldots, U_{Z, N+1}, V_{Z, 1}, V_{Z, 2}, \ldots, V_{Z, N+1}\right\} \\
& (j=1,2, \ldots, 2 N+2) .
\end{aligned}
$$

When $j=1,2, \ldots, N+1$

$$
F_{i, j}=Y_{i, j+N+1} \quad(i=1,2)
$$

and when $j=N+2, N+3, \ldots, 2 N+2$

$$
\begin{aligned}
& {\left[\begin{array}{c}
F_{1, j} \\
F_{2, j}
\end{array}\right]} \\
& =\left[\begin{array}{cc}
-2 \zeta & 2 \bar{\Omega} \\
-2 \bar{\Omega} & -2 \zeta
\end{array}\right]\left[\begin{array}{l}
Y_{1, j} \\
Y_{2, j}
\end{array}\right]+\left[\begin{array}{cc}
\bar{\Omega}^{2} & 0 \\
0 & \bar{\Omega}^{2}
\end{array}\right]\left[\begin{array}{l}
Y_{1, j-N-1} \\
Y_{2, j-N-1}
\end{array}\right] \\
& \quad-\left(\frac{1}{\pi^{4}} \frac{\partial^{4}}{\partial X^{4}}+\frac{\alpha+\beta \cos \left(Z_{p} \bar{\Omega} \tau\right)}{\pi^{2}} \frac{\partial^{2}}{\partial X^{2}}\right)\left[\begin{array}{l}
Y_{1, j-N-1} \\
Y_{2, j-N-1}
\end{array}\right] .
\end{aligned}
$$

Then, we have a unified semidiscretized equation:

$$
\frac{\partial Y_{i, j}}{\partial \tau}=F_{i, j} \quad(i=1,2, j=1,2, \ldots, 2 N+2) .
$$

The temporal discretization expressions of (16) by using fourth-order Runge-Kutta method are given as

$$
\begin{array}{r}
Y_{i, j}^{n+1}=Y_{i, j}^{n}+\Delta \tau Y_{i, j+N+1}^{n} \\
+\frac{\Delta \tau^{2}}{6}\left(L_{i, j+N+1,1}+L_{i, j+N+1,2}+L_{i, j+N+1,3}\right) \\
\quad(i=1,2, j=1,2, \ldots, N+1), \\
Y_{i, j}^{n+1}=Y_{i, j}^{n}+\frac{\Delta \tau}{6}\left(L_{i, j, 1}+2 L_{i, j, 2}+2 L_{i, j, 3}+L_{i, j, 4}\right) \\
\quad(i=1,2, \quad j=N+2, N+3, \ldots, 2 N+2),
\end{array}
$$

where $L_{i, j, 1}=F_{i, j, 1}^{n}, L_{i, j, 2}=F_{i, j, 2}^{n}, L_{i, j, 3}=F_{i, j, 3}^{n}$, and $L_{i, j, 4}=$ $F_{i, j, 4}^{n}(i=1,2, j=N+2, N+3, \ldots, 2 N+2)$ and here superscript $n$ denotes time level; $\Delta \tau$ is the time step, so $\tau=$ 
$n \Delta \tau$. Using the DSC discrete scheme (6), the $F_{i, j, 1}^{n}, F_{i, j, 2}^{n}, F_{i, j, 3}^{n}$, and $F_{i, j, 4}^{n}$ discretization expressions are

$$
\begin{aligned}
& {\left[\begin{array}{l}
L_{1, j, 1} \\
L_{2, j, 1}
\end{array}\right]} \\
& =\left[\begin{array}{l}
F_{1, j, 1}^{n} \\
F_{2, j, 1}^{n}
\end{array}\right]=\left[\begin{array}{cc}
-2 \zeta & 2 \bar{\Omega} \\
-2 \bar{\Omega} & -2 \zeta
\end{array}\right]\left[\begin{array}{l}
Y_{1, j}^{n} \\
Y_{2, j}^{n}
\end{array}\right] \\
& +\left[\begin{array}{cc}
\bar{\Omega}^{2} & 0 \\
0 & \bar{\Omega}^{2}
\end{array}\right]\left[\begin{array}{l}
Y_{1, j-N-1}^{n} \\
Y_{2, j-N-1}^{n}
\end{array}\right] \\
& -\left(\frac{1}{(\lambda l)^{4}} \sum_{k=-W}^{+W} \delta_{\Delta, \sigma}^{(4)}(-k \Delta X)\right. \\
& \left.+\frac{\left(\alpha+\beta \cos \left(Z_{p} \bar{\Omega} \tau\right)\right)}{(\lambda l)^{4}} \frac{\pi^{2}}{\eta^{2}} \sum_{k=-W}^{+W} \delta_{\Delta, \sigma}^{(2)}(-k \Delta X)\right) \\
& \cdot\left[\begin{array}{c}
Y_{1, j+k-N-1}^{n} \\
Y_{2, j+k-N-1}^{n}
\end{array}\right] \text {, } \\
& {\left[\begin{array}{l}
L_{1, j, 2} \\
L_{2, j, 2}
\end{array}\right]} \\
& =\left[\begin{array}{c}
F_{1, j, 2}^{n} \\
F_{2, j, 2}^{n}
\end{array}\right]=\left[\begin{array}{cc}
-2 \zeta & 2 \bar{\Omega} \\
-2 \bar{\Omega} & -2 \zeta
\end{array}\right]\left[\begin{array}{c}
Y_{1, j}^{n}+\left(\frac{\Delta \tau}{2}\right) L_{1, j, 1} \\
Y_{2, j}^{n}+\left(\frac{\Delta \tau}{2}\right) L_{2, j, 1}
\end{array}\right] \\
& +\left[\begin{array}{cc}
\bar{\Omega}^{2} & 0 \\
0 & \bar{\Omega}^{2}
\end{array}\right]\left[\begin{array}{l}
Y_{1, j-N-1}^{n}+\left(\frac{\Delta \tau}{2}\right) Y_{1, j}^{n} \\
Y_{2, j-N-1}^{n}+\left(\frac{\Delta \tau}{2}\right) Y_{2, j}^{n}
\end{array}\right] \\
& -\left(\frac{1}{(\lambda l)^{4}} \sum_{k=-W}^{+W} \delta_{\Delta, \sigma}^{(4)}(-k \Delta X)+\frac{\left(\alpha+\beta \cos \left(Z_{p} \bar{\Omega} \tau\right)\right)}{(\lambda l)^{4}} \frac{\pi^{2}}{\eta^{2}}\right. \\
& \left.\cdot \sum_{k=-W}^{+W} \delta_{\Delta, \sigma}^{(2)}(-k \Delta X)\right)\left[\begin{array}{l}
Y_{1, j+k-N-1}^{n}+\left(\frac{\Delta \tau}{2}\right) Y_{1, j+k}^{n} \\
Y_{2, j+k-N-1}^{n}+\left(\frac{\Delta \tau}{2}\right) Y_{2, j+k}^{n}
\end{array}\right] \\
& {\left[\begin{array}{l}
L_{1, j, 3} \\
L_{2, j, 3}
\end{array}\right]} \\
& =\left[\begin{array}{c}
F_{1, j, 3}^{n} \\
F_{2, j, 3}^{n}
\end{array}\right]=\left[\begin{array}{cc}
-2 \zeta & 2 \bar{\Omega} \\
-2 \bar{\Omega} & -2 \zeta
\end{array}\right]\left[\begin{array}{l}
Y_{1, j}^{n}+\left(\frac{\Delta \tau}{2}\right) L_{1, j, 2} \\
Y_{2, j}^{n}+\left(\frac{\Delta \tau}{2}\right) L_{2, j, 2}
\end{array}\right] \\
& +\left[\begin{array}{cc}
\bar{\Omega}^{2} & 0 \\
0 & \bar{\Omega}^{2}
\end{array}\right]\left[\begin{array}{l}
Y_{1, j-N-1}^{n}+\left(\frac{\Delta \tau}{2}\right) Y_{1, j}^{n}+\left(\frac{\Delta \tau^{2}}{4}\right) L_{1, j, 1} \\
Y_{2, j-N-1}^{n}+\left(\frac{\Delta \tau}{2}\right) Y_{2, j}^{n}+\left(\frac{\Delta \tau^{2}}{4}\right) L_{2, j, 1}
\end{array}\right]
\end{aligned}
$$

$$
\begin{aligned}
& -\left(\frac{1}{(\lambda l)^{4}} \sum_{k=-W}^{+W} \delta_{\Delta, \sigma}^{(4)}(-k \Delta X)+\frac{\left(\alpha+\beta \cos \left(Z_{p} \bar{\Omega} \tau\right)\right)}{(\lambda l)^{4}} \frac{\pi^{2}}{\eta^{2}}\right. \\
& \left.\cdot \sum_{k=-W}^{+W} \delta_{\Delta, \sigma}^{(2)}(-k \Delta X)\right) \\
& {\left[\begin{array}{c}
Y_{1, j+k-N-1}^{n}+\left(\frac{\Delta \tau}{2}\right) Y_{1, j+k}^{n}+\left(\frac{\Delta \tau^{2}}{4}\right) L_{1, j+k, 1} \\
Y_{2, j+k-N-1}^{n}+\left(\frac{\Delta \tau}{2}\right) Y_{2, j+k}^{n}+\left(\frac{\Delta \tau^{2}}{4}\right) L_{2, j+k, 1}
\end{array}\right]} \\
& {\left[\begin{array}{l}
L_{1, j, 4} \\
L_{2, j, 4}
\end{array}\right]} \\
& =\left[\begin{array}{l}
F_{1, j, 4}^{n} \\
F_{2, j, 4}^{n}
\end{array}\right]=\left[\begin{array}{cc}
-2 \zeta & 2 \bar{\Omega} \\
-2 \bar{\Omega} & -2 \zeta
\end{array}\right]\left[\begin{array}{l}
Y_{1, j}^{n}+\Delta \tau L_{1, j, 3} \\
Y_{2, j}^{n}+\Delta \tau L_{2, j, 3}
\end{array}\right] \\
& +\left[\begin{array}{cc}
\bar{\Omega}^{2} & 0 \\
0 & \bar{\Omega}^{2}
\end{array}\right]\left[\begin{array}{c}
Y_{1, j-N-1}^{n}+\Delta \tau Y_{1, j}^{n}+\left(\frac{\Delta \tau^{2}}{2}\right) L_{1, j, 2} \\
Y_{2, j-N-1}^{n}+\Delta \tau Y_{2, j}^{n}+\left(\frac{\Delta \tau^{2}}{2}\right) L_{2, j, 2}
\end{array}\right] \\
& -\left(\frac{1}{(\lambda l)^{4}} \sum_{k=-W}^{+W} \delta_{\Delta, \sigma}^{(4)}(-k \Delta X)+\frac{\left(\alpha+\beta \cos \left(Z_{p} \bar{\Omega} \tau\right)\right)}{(\lambda l)^{4}} \frac{\pi^{2}}{\eta^{2}}\right. \\
& \left.\cdot \sum_{k=-W}^{+W} \delta_{\Delta, \sigma}^{(2)}(-k \Delta X)\right) \\
& \cdot\left[\begin{array}{l}
Y_{1, j+k-N-1}^{n}+\Delta \tau Y_{1, j+k}^{n}+\left(\frac{\Delta \tau^{2}}{2}\right) L_{1, j+k, 2} \\
Y_{2, j+k-N-1}^{n}+\Delta \tau Y_{2, j+k}^{n}+\left(\frac{\Delta \tau^{2}}{2}\right) L_{2, j+k, 2}
\end{array}\right] \\
& (j=N+2, N+3, \ldots, 2 N+2),
\end{aligned}
$$

where $[-W,+W]$ is the computational bandwidth. Kernels $\delta_{\Delta, \sigma}^{(2)}$ and $\delta_{\Delta, \sigma}^{(4)}$ can be easily obtained [20,23]. All of these coefficients are only dependent on grid size. When the grid point distribution is given, the coefficients can be computed once and stored for use during the computation.

The overall calculation procedure can be summarized as follows $[39,40]$ :

(a) the initial values for $Y_{i, j}^{0}$ or the values of $Y_{i, j}^{n}(i=$ $1,2, j=1,2, \ldots, 2 N+2)$ at previous time level $n$ can be obtained;

(b) the values of $F_{i, j, 1}^{n}, F_{i, j, 2}^{n}, F_{i, j, 3}^{n}$, and $F_{i, j, 4}^{n}(i=1,2, j=$ $N+2, N+3, \ldots, 2 N+2)$ at the time level $n$ can be obtained;

(c) substituting the values in (18) into (17), the values of $Y_{i, j}^{n+1}(i=1,2, j=1,2, \ldots, 2 N+2)$ at new time level $n+1$ can be calculated; 


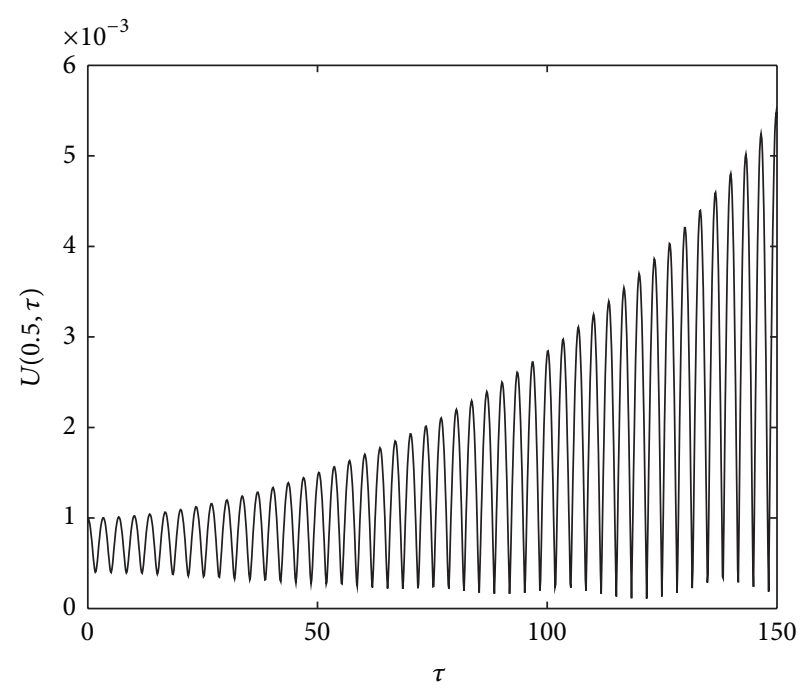

(a)

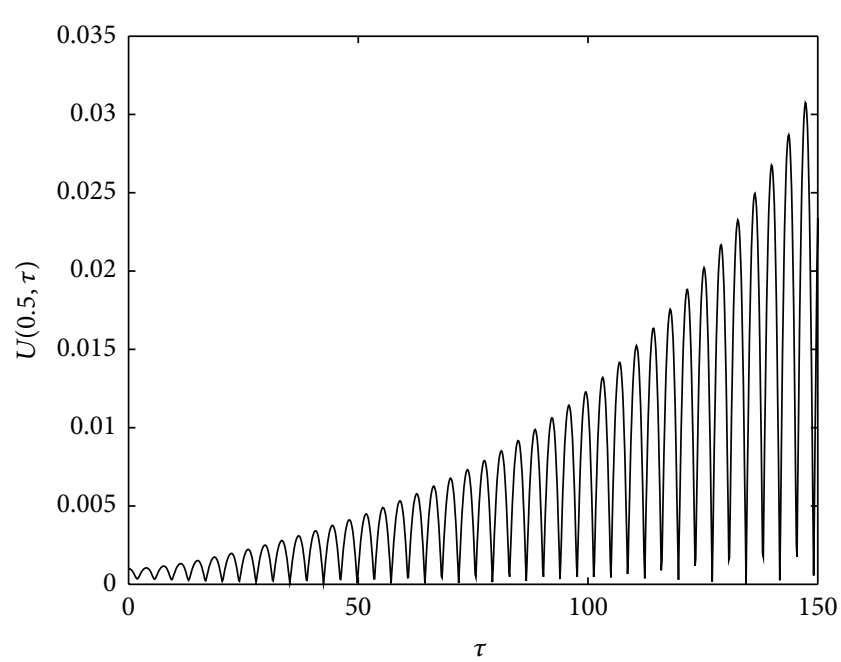

(c)

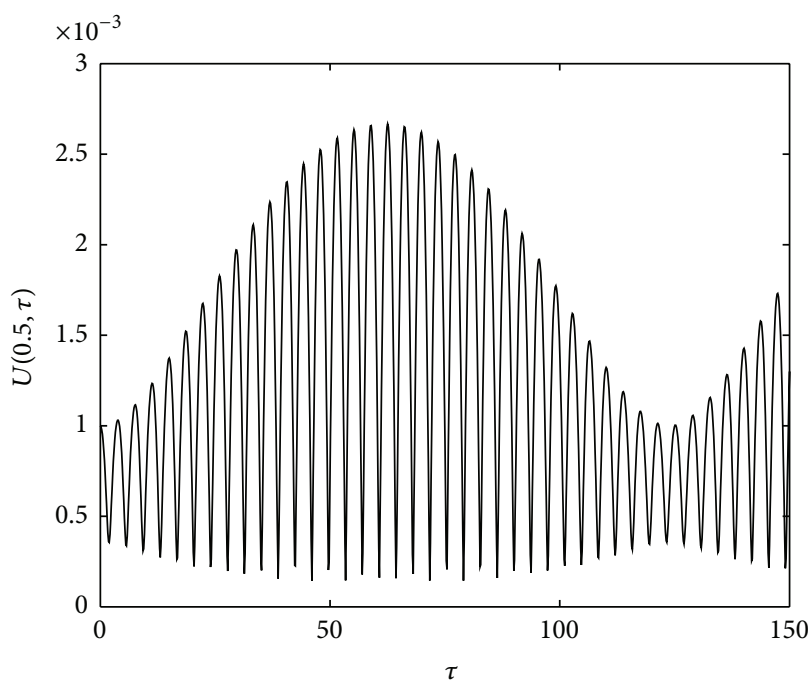

(b)

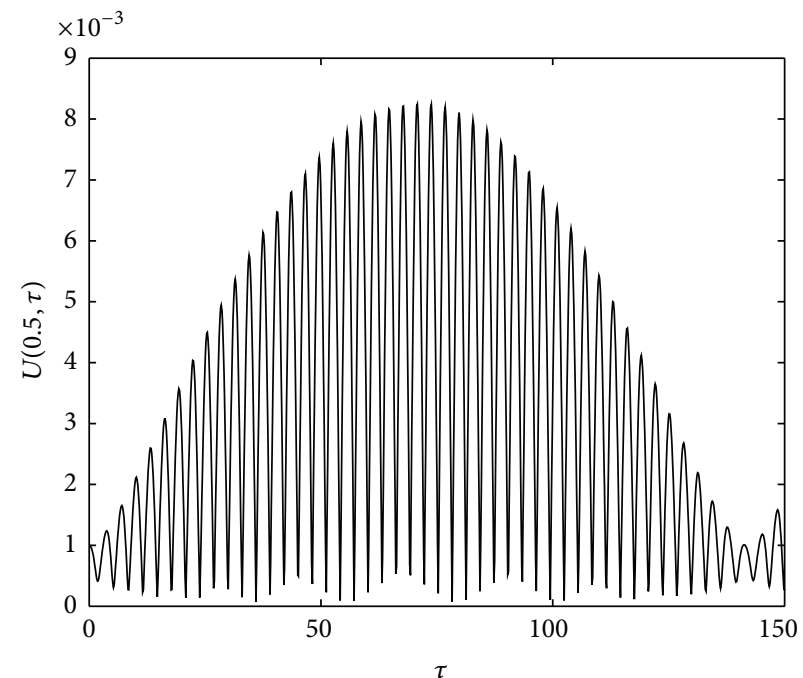

(d)

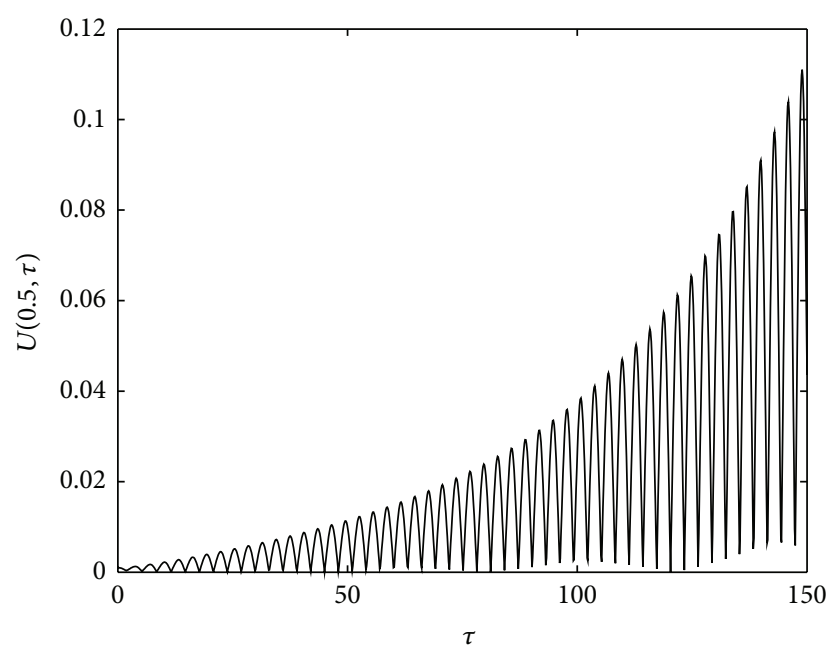

(e)

FIGURE 2: Dynamic responses of transverse displacements with $Z_{p}=5$. (a) $Z_{p} \Omega / 2 \omega^{*}=1.0 \beta=0.05$, (b) $Z_{p} \Omega / 2 \omega^{*}=0.9 \beta=0.34$, (c) $Z_{p} \Omega / 2 \omega^{*}=0.9 \beta=0.36$, (d) $Z_{p} \Omega / 2 \omega^{*}=1.1 \beta=0.36$, and (e) $Z_{p} \Omega / 2 \omega^{*}=1.1 \beta=0.38$. 


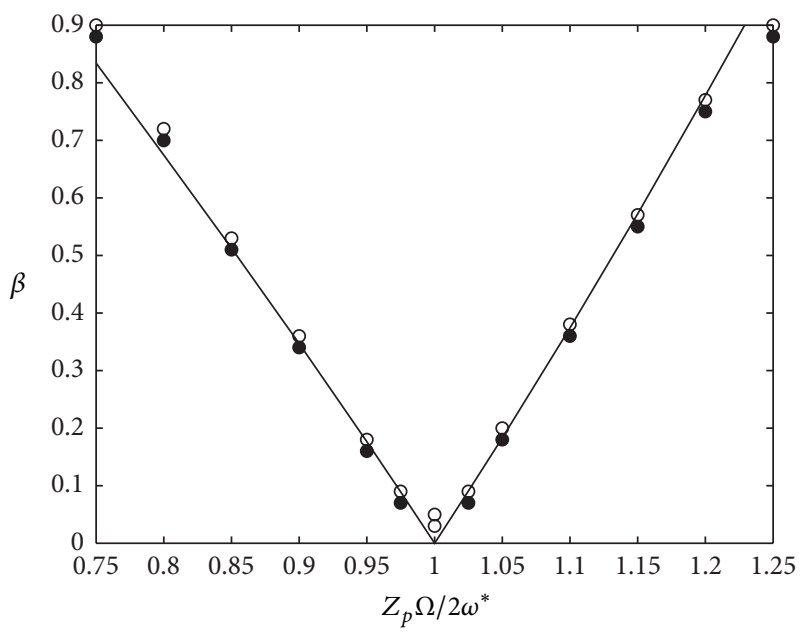

FIGURE 3: Dynamic instability region of a rotating ship shaft with $Z_{p}$ $=5$. "-," instability region boundary by Floquet's method; "๑" and "O," stable and unstable results by DSC method.

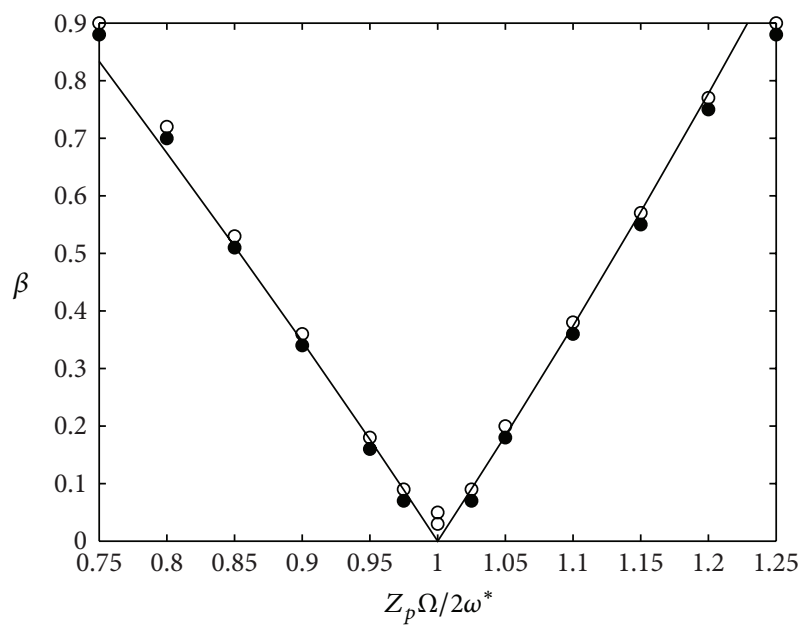

FIGURE 4: Dynamic instability region of a rotating ship shaft with $Z_{p}=3$. Key as for Figure 3 .

(d) the computational time is advanced (i.e., $\tau=\tau+\Delta \tau$, $n=n+1)$, and the whole procedure above is repeated, until calculation precision is reached.

\section{Numerical Results and Discussions}

4.1. Dynamic Instability Regions for Shafts without Damping. In this section, first, dynamic instability regions with different number of blades $Z_{p}$ are carried out. And then effect of constant term in the periodic axial force on dynamic instability region is discussed. In order to discuss the effects of number of blades $Z_{p}$ on dynamic instability regions, the value of $\alpha$ is chosen as 0.1. Then the frequency is $\omega^{*}=\omega \sqrt{1-\alpha}=\sqrt{0.9} \omega$. The initial conditions and parameters in DSC algorithm are set as follows:

$U_{Y}(i \Delta X, 0)=0.001 \sin (i \Delta X \pi), U_{Z}(i \Delta X, 0)=0$, $\partial U_{Y}(i \Delta X, 0) / \partial \tau=0, \partial U_{Z}(i \Delta X, 0) / \partial \tau=0(i=0,1,2, \ldots, N)$,

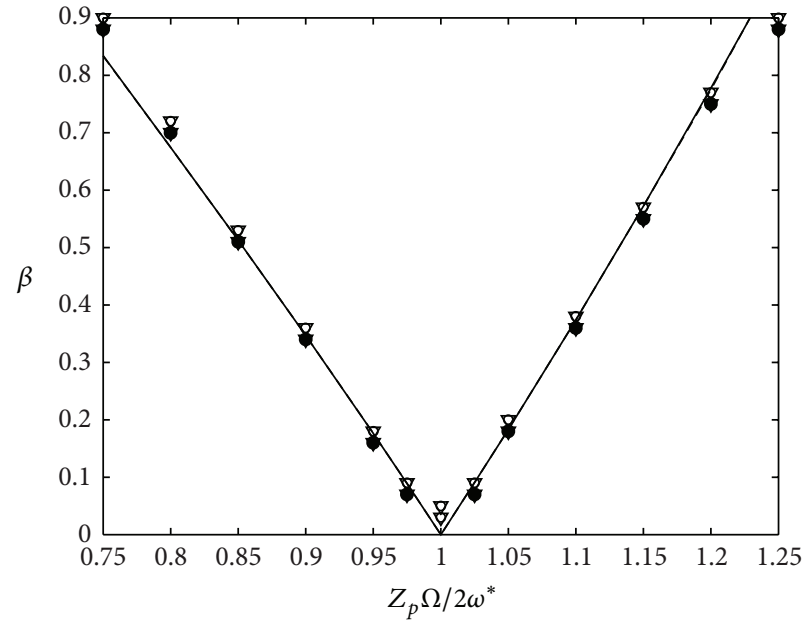

FIGURE 5: Dynamic instability regions with different number of blades $Z_{p}$. "-," “- -," instability region boundary with $Z_{p}=5$, $Z_{p}=3$ by Floquet's method; “” "O," " $\nabla$ " " $\nabla$," stable and unstable results with $Z_{p}=5, Z_{p}=3$ by DSC method.

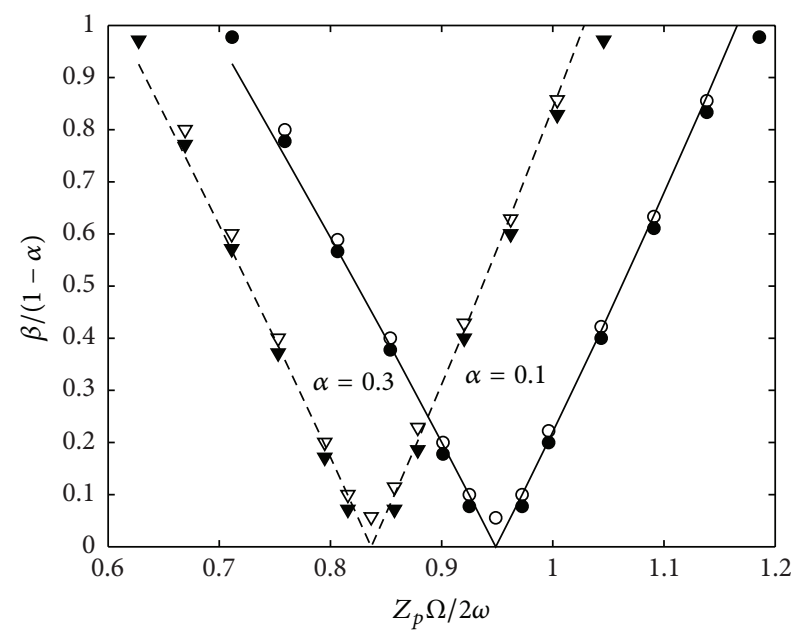

Figure 6: Dynamic instability regions with different $\alpha$. “-," “- -," instability region boundary with $\alpha=0.1,0.3$ by Floquet's method; “๑” "O," " $\nabla$ " “ $\nabla$," stable and unstable results with $\alpha=0.1,0.3$ by DSC method.

$N=16, W=15, r=2.5, \Delta \tau=3.0 \times 10^{-6}$. For the choice of $N, W$, and $r$, readers may refer to Wei et al. [20,26].

Figure 2 shows dynamic responses of transverse dimensionless displacements at midpoint for the rotating shaft with $Z_{p}=5$ and $\zeta=0$. It is seen that the dynamic responses for $Z_{p} \Omega / 2 \omega^{*}=1.0 \beta=0.05, Z_{p} \Omega / 2 \omega^{*}=0.9 \beta=0.36$, and $Z_{p} \Omega / 2 \omega^{*}=1.1 \beta=0.38$ in Figures $2(\mathrm{a}), 2(\mathrm{c})$, and $2(\mathrm{e})$ are dynamically unstable, while the dynamic responses for $Z_{p} \Omega / 2 \omega^{*}=0.9 \beta=0.34$ and $Z_{p} \Omega / 2 \omega^{*}=1.1 \beta=0.36$ in Figures $2(\mathrm{~b})$ and $2(\mathrm{~d})$ are dynamically stable. The whole procedure above is repeated, the dynamic instability region for the first mode shown as Figure 3 is obtained. In Figure 3, the dynamic instability region obtained by DSC algorithm is coincident with the instability region obtained by Floquet's 


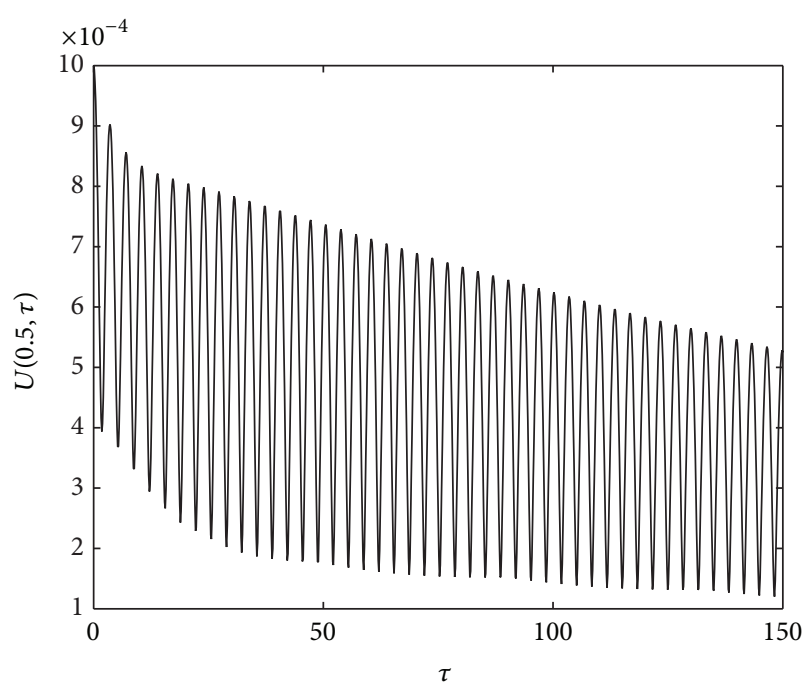

(a)

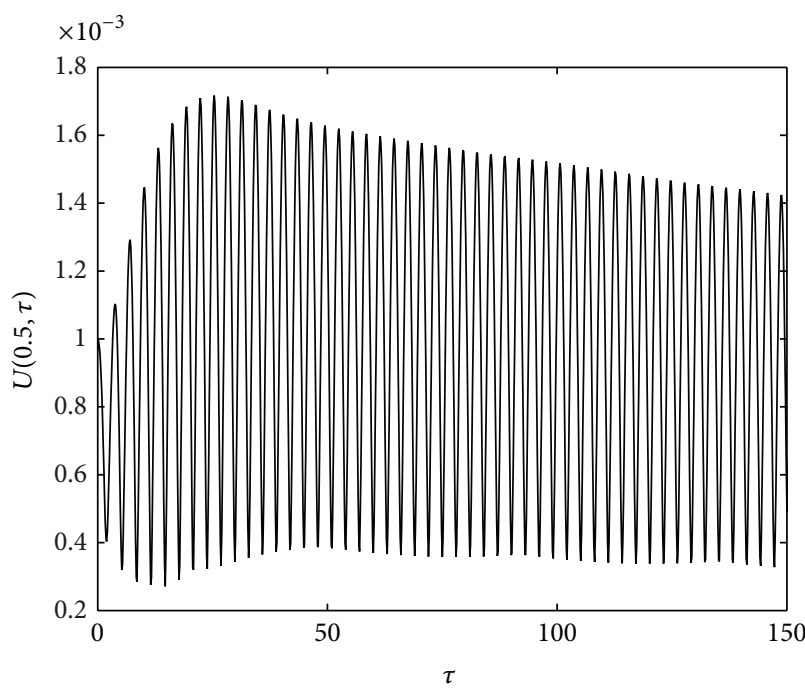

(c)

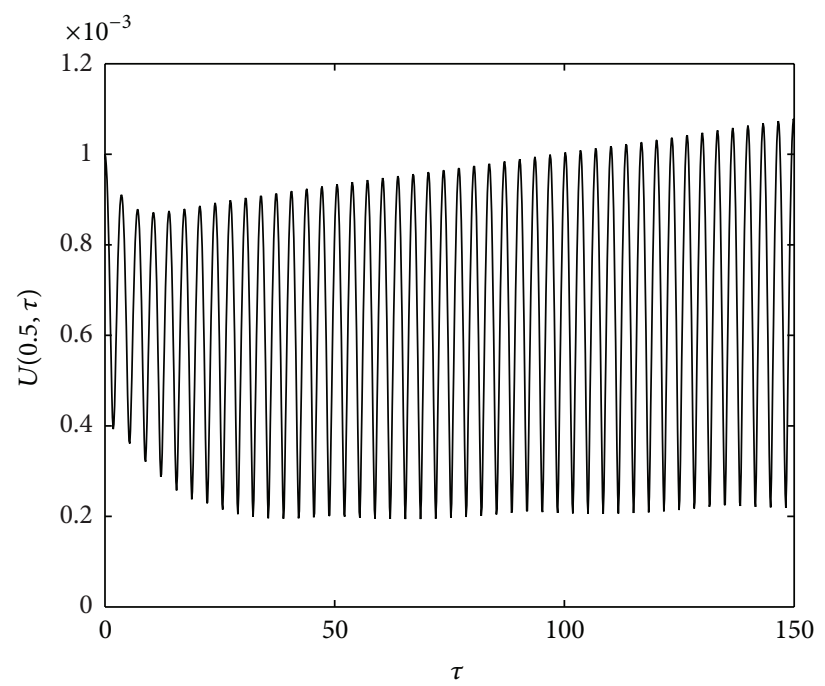

(b)

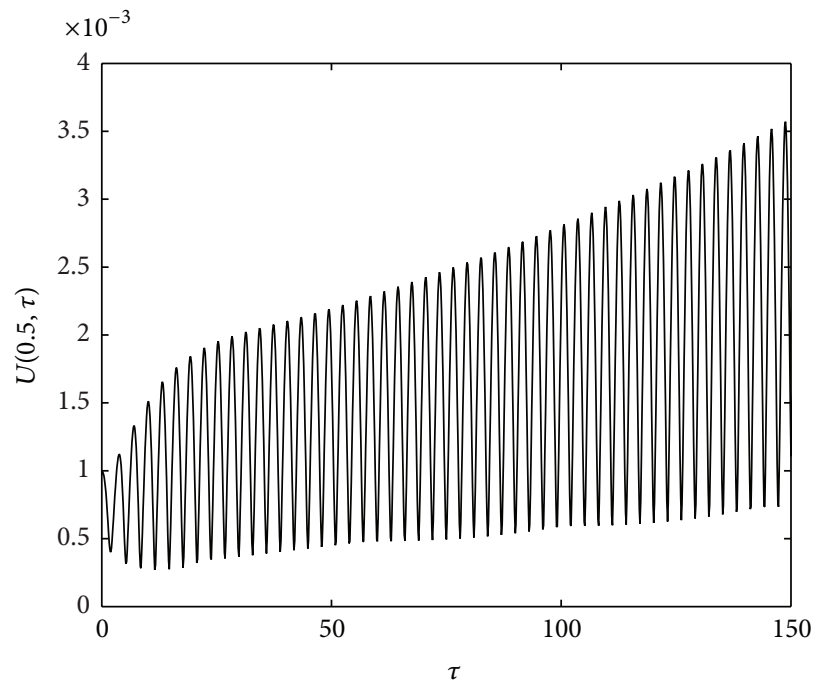

(d)

FIGURE 7: Dynamic responses of transverse displacements with $Z_{p}=5$ and $\zeta=0.05$. (a) $Z_{p} \Omega / 2 \omega^{*}=1.0 \beta=0.16$, (b) $Z_{p} \Omega / 2 \omega^{*}=1.0 \beta=$ 0.18 , (c) $Z_{p} \Omega / 2 \omega^{*}=1.1 \beta=0.38$, and (d) $Z_{p} \Omega / 2 \omega^{*}=1.1 \beta=0.40$.

method [15] very well. It is also found that the dynamic instability region is " $V$ " shape, and when $Z_{p} \Omega / 2 \omega^{*}=1.0$, that is to say, the excitation frequency is twice the natural frequency with constant axial compressive force, the dynamic instability is the most serious, which is similar to the dynamic instability region when a nonspinning shaft is subjected to periodic force [1].

The dynamic instability region with $Z_{p}=3$ shown as Figure 4 is also obtained by using DSC algorithm. It is found that the dynamic instability region is " $V$ " shape, and when $Z_{p} \Omega / 2 \omega^{*}=1.0$, the dynamic instability is the most serious. And also the instability region obtained by DSC algorithm agrees well with that obtained by Floquet's method. The comparisons of dynamic instability regions with different number of blades $Z_{p}$ are shown in Figure 5. It is seen that the dynamic instability regions obtained by DSC algorithm and Floquet's method are " $V$ " shapes and do not change with the number of blades $Z_{p}$ increasing. That is to say, the increase of number of blades $Z_{p}$ has little influence on dynamic instability region of rotating ship shafts without damping.

In order to discuss the effect of constant term in the periodic force on dynamic instability region, the value of $\alpha$ is increased to 0.3 . And the corresponding frequency $\omega^{*}=\omega \sqrt{1-\alpha}=\sqrt{0.7} \omega=0.8367 \omega$. When $\alpha=0.1$, the corresponding frequency is $\omega^{*}=\sqrt{0.9} \omega=0.9487 \omega$. The comparisons of dynamic instability regions with different $\alpha$ are shown in Figure 6. It is found that the dynamic instability regions with $\alpha=0.1$ and 0.3 are " $V$ " shape. And when the excitation frequency $Z_{p} \Omega$ is near twice the natural frequency with constant axial compressive force, the dynamic instability is the most serious. The increase in the value of $\alpha$ causes 
the dynamic instability regions shifting to lower exciting frequencies, which makes the shaft more sensitive to periodic force.

4.2. Dynamic Instability Regions for Shafts with Damping. In this section, dynamic instability regions with damping effects are obtained, and effects of different number of blades $Z_{p}$ are also discussed. The value of $\zeta$ is chosen as 0.05 . And the value of $\alpha$ is chosen as 0.1 . The initial conditions and other parameters in DSC algorithm are the same as the previous computation.

Dynamic responses of transverse dimensionless displacements at midpoint for the rotating shaft with $Z_{p}=5$ and $\zeta=0.05$ are shown in Figure 7. It is seen that the dynamic responses for $Z_{p} \Omega / 2 \omega^{*}=1.0 \beta=0.18$ and $Z_{p} \Omega / 2 \omega^{*}=1.1$ $\beta=0.40$ in Figures $7(\mathrm{~b})$ and $7(\mathrm{~d})$ are dynamically unstable, while the dynamic responses for $Z_{p} \Omega / 2 \omega^{*}=1.0 \beta=0.16$ and $Z_{p} \Omega / 2 \omega^{*}=1.1 \beta=0.38$ in Figures $7(\mathrm{a})$ and $7(\mathrm{c})$ are dynamically stable. The whole procedure above is repeated; the dynamic instability region shown in Figure 8 is obtained. In Figure 8 , the dynamic instability region obtained by DSC algorithm is coincident with that obtained by Floquet's method very well. And it is also found that damping improves dynamic stability of the region with $Z_{p} \Omega / 2 \omega^{*}$ near 1.0 obviously.

The dynamic instability region with $Z_{p}=3$ and $\zeta=0.05$ shown in Figure 9 is also obtained by using DSC algorithm. It is found that the dynamic stability of the region with $Z_{p} \Omega / 2 \omega^{*}$ near 1.0 is improved obviously due to the existent of damping, while dynamic stability of other regions decreases. The comparisons of dynamic instability regions with different number of blades $Z_{p}$ and $\zeta=0.05$ are shown in Figure 10. It is also seen that the dynamic instability regions obtained by DSC algorithm agree well with those obtained by Floquet's method, respectively. And the dynamic instability regions expand with decreasing of the number of blades $Z_{p}$; that is to say, the more blades on the propeller, the better of dynamic stability, which is different from that obtained from Figure 5. Moreover, the dynamic instability of region with $Z_{p} \Omega / 2 \omega^{*}$ near 1.0 is also the most serious, although the dynamic stability of this region is better than that shown in Figure 5. Therefore, in order to prevent dynamic instability occurring, the spinning speed $\Omega$ should be far away from $2 \omega^{*} / Z_{p}$.

\section{Conclusions}

In the present study, the method of discrete singular convolution (DSC) has been applied to solve vibration governing equations of motion for rotating ship shafts under periodic axial forces with type of $P(t)=P_{S}+P_{D} \cos \left(Z_{p} \Omega t\right)$. The excitation frequency of the periodic axial force is related to the spinning speed and the number of blades. The dynamic instability regions of rotating ship shafts are obtained through judging the dynamic stability of dynamic responses of transverse displacements. Effects of number of blades, constant

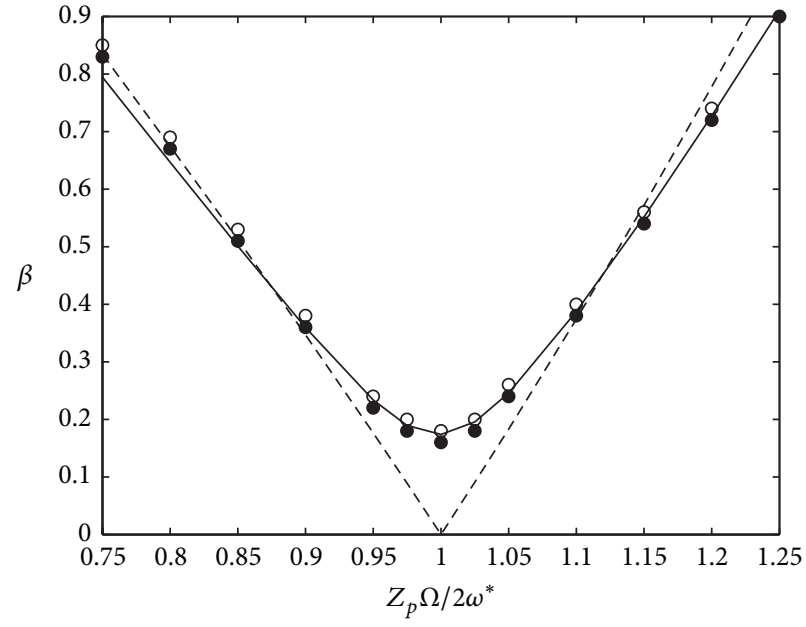

FIGURE 8: Dynamic instability region of a rotating ship shaft with $Z_{p}$ $=5$ and $\zeta=0.05$. "-," " “- -," instability region boundary with $Z_{p}=5$ $\zeta=0.05$ and $Z_{p}=5 \zeta=0$ by Floquet's method, "@," "O," stable and unstable data with $Z_{p}=5 \zeta=0.05$ by DSC method.

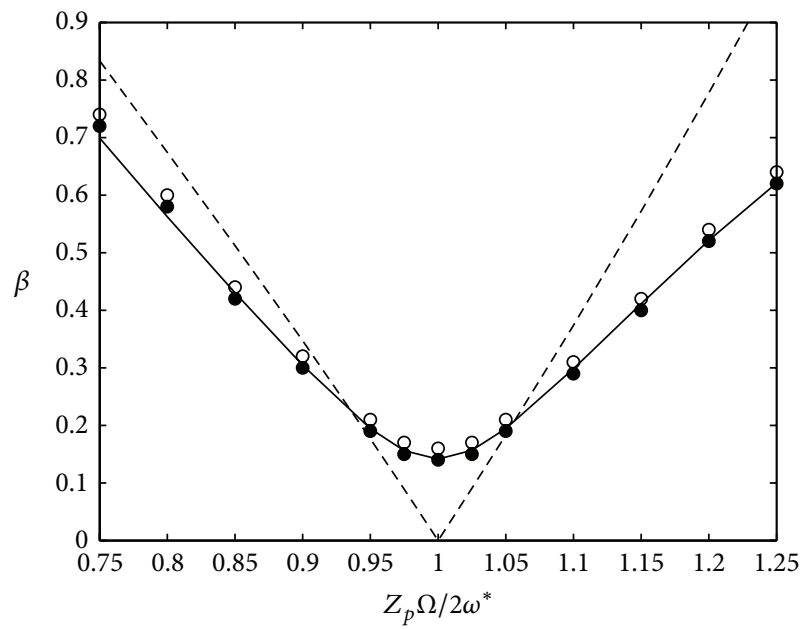

FIGURE 9: Dynamic instability region of a rotating ship shaft with $Z_{p}$ $=3$ and $\zeta=0.05$. “-," " “- -, instability region boundary with $Z_{p}=3$, $\zeta=0.05$ and $Z_{p}=3, \zeta=0$ by Floquet's method; " $\bullet$," "O," stable and unstable data with $Z_{p}=3, \zeta=0.05$ by DSC method.

term in the periodic force, and damping on dynamic instability regions also have been discussed. The following can be concluded.

(1) The dynamic instability regions for different cases obtained by using DSC method are coincident with the instability regions obtained by Floquet's method very well, respectively, which verifies the application of DSC method to this class of problems.

(2) For the rotating ship shaft without damping, the dynamic instability regions are " $V$ " shape and do not change with the number of blades increasing. The increase in the value of the constant term in the periodic force causes the dynamic instability regions 


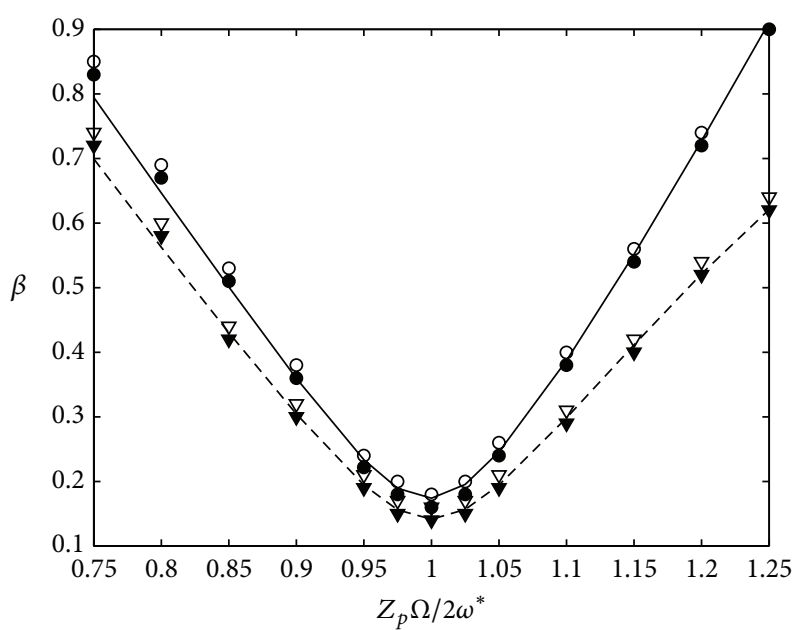

FIGURE 10: Dynamic instability regions with different numbers of blades $Z_{p}$ and $\zeta=0.05$. “-," "- -," instability region boundary with $Z_{p}=5, Z_{p}=3$ by Floquet's method, “” “O," “ $\nabla$ " “ $\nabla$," stable and unstable data with $Z_{p}=5, Z_{p}=3$ by DSC method.

shifting to lower exciting frequencies, which makes the shaft more sensitive to periodic force.

(3) For the rotating ship shaft with damping, the dynamic stability of regions where the excitation frequency is in the neighborhood of twice the natural frequency with constant axial compressive force is improved obviously, due to damping existing, while the dynamic stability of other regions decrease. In addition, the dynamic instability regions expand with the number of blades decreasing; that is to say, the increase of number of blades improves the dynamic stability, which is very different from the result for the rotating ship shaft without damping. Therefore, it is suggested that the screw propeller should have more blades for rotating ship shafts.

(4) When the excitation frequency is twice the natural frequency with constant axial compressive force, the dynamic instability is the most serious for the system with no damping and with damping. Therefore, the spinning speed $\Omega$ should be far away from $2 \omega^{*} / Z_{p}$, to avoid serious dynamic instability happening.

\section{Conflict of Interests}

The authors declare that there is no conflict of interests regarding the publication of this paper.

\section{References}

[1] V. V. Bolotin, The Dynamic Stability of Elastic Systems, HoldenDay Inc, New York, NY, USA, 1965.

[2] L. W. Chen and D. M. Ku, "Dynamic stability analysis of a rotating shaft by the finite element method," Journal of Sound and Vibration, vol. 143, no. 1, pp. 143-151, 1990.
[3] L.-W. Chen and D.-M. Ku, "Dynamic stability of a cantilever shaft-disk system," Journal of Vibration and Acoustics, Transactions of the ASME, vol. 114, no. 3, pp. 326-329, 1992.

[4] H. P. Lee, "Dynamic stability of spinning pre-twisted beams subject to axial pulsating loads," Computer Methods in Applied Mechanics and Engineering, vol. 127, no. 1-4, pp. 115-126, 1995.

[5] H. P. Lee, "Effects of axial base excitations on the dynamic stability of spinning per-twisted cantilever beams," Journal of Sound and Vibration, vol. 185, no. 2, pp. 265-278, 1995.

[6] H. P. Lee, T. H. Tan, and G. S. B. Leng, "Dynamic stability of spinning Timoshenko shafts with a time-dependent spin rate," Journal of Sound and Vibration, vol. 199, no. 3, pp. 401-415, 1997.

[7] T. H. Tan, H. P. Lee, and G. S. B. Leng, "Parametric instability of spinning pretwisted beams subjected to spin speed perturbation," Computer Methods in Applied Mechanics and Engineering, vol. 148, no. 1-2, pp. 139-163, 1997.

[8] C.-L. Liao and B.-W. Huang, "Parametric instability of a spinning pretwisted beam under periodic axial force," International Journal of Mechanical Sciences, vol. 37, no. 4, pp. 423-439, 1995.

[9] C.-L. Liao and B.-W. Huang, "Parametric resonance of a spinning pretwisted beam with time-dependent spinning rate," Journal of Sound and Vibration, vol. 180, no. 1, pp. 47-65, 1995.

[10] L.-W. Chen and W.-K. Peng, "Dynamic stability of rotating composite shafts under periodic axial compressive loads," Journal of Sound and Vibration, vol. 212, no. 2, pp. 215-230, 1998.

[11] H. C. Sheu and L. W. Chen, "Lumped mass model for parametric instability analysis of cantilever shaft-disk systems," Journal of Sound and Vibration, vol. 234, no. 2, pp. 331-348, 2000.

[12] T. H. Young and C. Y. Gau, "Dynamic stability of spinning pretwisted beams subjected to axial random forces," Journal of Sound and Vibration, vol. 268, no. 1, pp. 149-165, 2003.

[13] T. H. Young and C. Y. Gau, "Dynamic stability of pre-twisted beams with non-constant spin rates under axial random forces," International Journal of Solids and Structures, vol. 40, no. 18, pp. 4675-4698, 2003.

[14] C.-Y. Lin and L.-W. Chen, "Dynamic stability of spinning pretwisted sandwich beams with a constrained damping layer subjected to periodic axial loads," Composite Structures, vol. 70, no. 3, pp. 275-286, 2005.

[15] Y.-C. Pei, "Stability boundaries of a spinning rotor with parametrically excited gyroscopic system," European Journal of Mechanics-A/Solids, vol. 28, no. 4, pp. 891-896, 2009.

[16] R. Pavlović, P. Kozić, S. Mitić, and I. Pavlović, "Stochastic stability of a rotating shaft," Archive of Applied Mechanics, vol. 79, no. 12, pp. 1163-1171, 2009.

[17] Z. Chen, Vibration of Marine Propulsion Shaft, Shanghai Jiao Tong University Press, Shanghai, China, 1987, (Chinese).

[18] G. W. Wei, "Discrete singular convolution for the solution of the Fokker-Planck equation," Journal of Chemical Physics, vol. 110, no. 18, pp. 8930-8942, 1999.

[19] G. W. Wei, "Vibration analysis by discrete singular convolution," Journal of Sound and Vibration, vol. 244, no. 3, pp. 535-553, 2001.

[20] G. W. Wei, "Discrete singular convolution for beam analysis," Engineering Structures, vol. 23, no. 9, pp. 1045-1053, 2001.

[21] G. W. Wei, Y. B. Zhao, and Y. Xiang, "A novel approach for the analysis of high-frequency vibrations," Journal of Sound and Vibration, vol. 257, no. 2, pp. 207-246, 2002.

[22] S. Zhao, G. W. Wei, and Y. Xiang, "DSC analysis of free-edged beams by an iteratively matched boundary method," Journal of Sound and Vibration, vol. 284, no. 1-2, pp. 487-493, 2005. 
[23] G. W. Wei, "A new algorithm for solving some mechanical problems," Computer Methods in Applied Mechanics and Engineering, vol. 190, no. 15-17, pp. 2017-2030, 2001.

[24] G. W. Wei, Y. B. Zhao, and Y. Xiang, "The determination of natural frequencies of rectangular plates with mixed boundary conditions by discrete singular convolution," International Journal of Mechanical Sciences, vol. 43, no. 8, pp. 1731-1746, 2001.

[25] Y. B. Zhao and G. W. Wei, "DSC analysis of rectangular plates with non-uniform boundary conditions," Journal of Sound and Vibration, vol. 255, no. 2, pp. 203-228, 2003.

[26] G. W. Wei, Y. B. Zhao, and Y. Xiang, "Discrete singular convolution and its application to the analysis of plates with internal supports. Part 1: Theory and algorithm," International Journal for Numerical Methods in Engineering, vol. 55, no. 8, pp. 913-946, 2002.

[27] Y. Xiang, Y. B. Zhao, and G. W. Wei, "Discrete singular convolution and its application to the analysis of plates with internal supports. II. Applications," International Journal for Numerical Methods in Engineering, vol. 55, no. 8, pp. 947-971, 2002.

[28] Y. B. Zhao, G. W. Wei, and Y. Xiang, "Discrete singular convolution for the prediction of high frequency vibration of plates," International Journal of Solids and Structures, vol. 39, no. 1, pp. 65-88, 2001.

[29] Y. B. Zhao, G. W. Wei, and Y. Xiang, "Plate vibration under irregular internal supports," International Journal of Solids and Structures, vol. 39, no. 5, pp. 1361-1383, 2002.

[30] Ö. Civalek, "An efficient method for free vibration analysis of rotating truncated conical shells," International Journal of Pressure Vessels and Piping, vol. 83, no. 1, pp. 1-12, 2006.

[31] Ö. Civalek, "Numerical analysis of free vibrations of laminated composite conical and cylindrical shells: discrete singular convolution (DSC) approach," Journal of Computational and Applied Mathematics, vol. 205, no. 1, pp. 251-271, 2007.

[32] Ö. Civalek, "A parametric study of the free vibration analysis of rotating laminated cylindrical shells using the method of discrete singular convolution," Thin-Walled Structures, vol. 45, no. 7-8, pp. 692-698, 2007.

[33] Ö. Civalek, "Fundamental frequency of isotropic and orthotropic rectangular plates with linearly varying thickness by discrete singular convolution method," Applied Mathematical Modelling, vol. 33, no. 10, pp. 3825-3835, 2009.

[34] C. W. Lim, Z. R. Li, and G. W. Wei, "DSC-Ritz method for highmode frequency analysis of thick shallow shells," International Journal for Numerical Methods in Engineering, vol. 62, no. 2, pp. 205-232, 2005.

[35] Y. Hou, G. W. Wei, and Y. Xiang, "DSC-Ritz method for the free vibration analysis of Mindlin plates," International Journal for Numerical Methods in Engineering, vol. 62, no. 2, pp. 262-288, 2005.

[36] Y. Xiang, S. K. Lai, and L. Zhou, "DSC-element method for free vibration analysis of rectangular Mindlin plates," International Journal of Mechanical Sciences, vol. 52, no. 4, pp. 548-560, 2010.

[37] Y. Xiang, S. K. Lai, L. Zhou, and C. W. Lim, "DSC-Ritz element method for vibration analysis of rectangular Mindlin plates with mixed edge supports," European Journal of Mechanics, A/Solids, vol. 29, no. 4, pp. 619-628, 2010.

[38] X. W. Wang, C. L. Xu, and S. M. Xu, "The discrete singular convolution for analyses of elastic wave propagations in onedimensional structures," Applied Mathematical Modelling, vol. 34, no. 11, pp. 3493-3508, 2010.
[39] Z. W. Song, W. Li, and G. R. Liu, "Stability and non-stationary vibration analysis of beams subjected to periodic axial forces using discrete singular convolution," Structural Engineering and Mechanics, vol. 44, no. 4, pp. 487-499, 2012.

[40] D. C. Wan and G. W. Wei, "The study of quasi wavelets based numerical method applied to Burgers' equations," Applied Mathematics and Mechanics (English Edition), vol. 21, no. 10, pp. 1099-1110, 2000. 

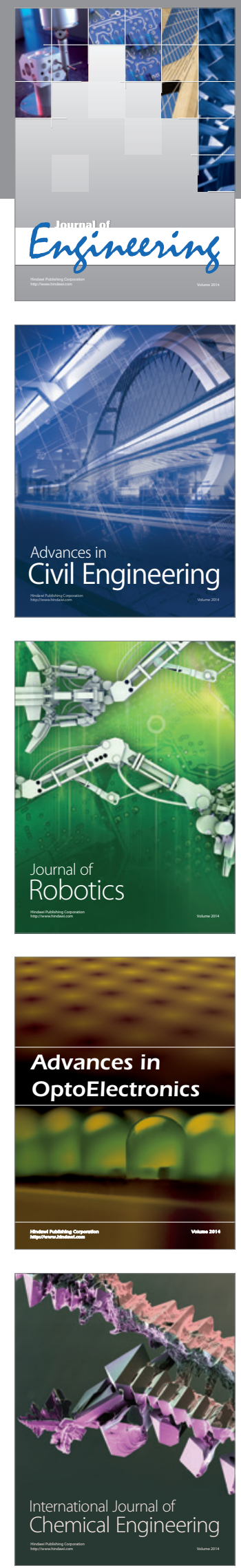

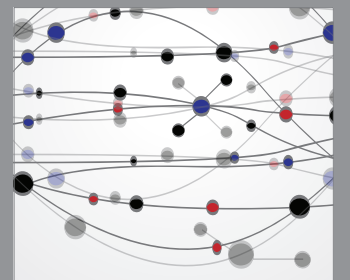

The Scientific World Journal
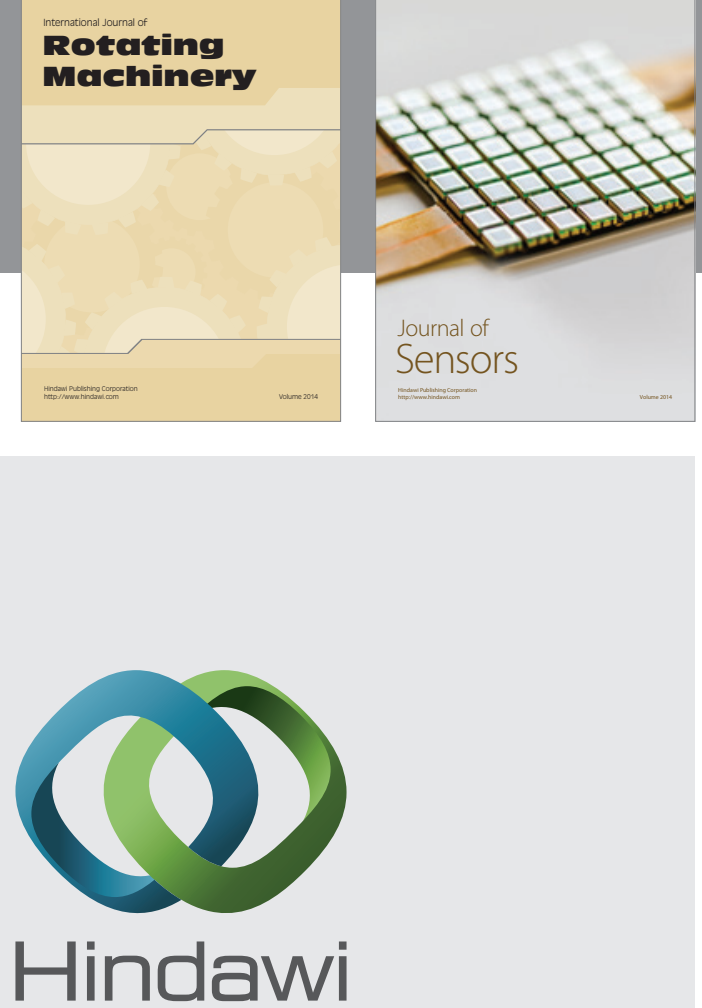

Submit your manuscripts at http://www.hindawi.com
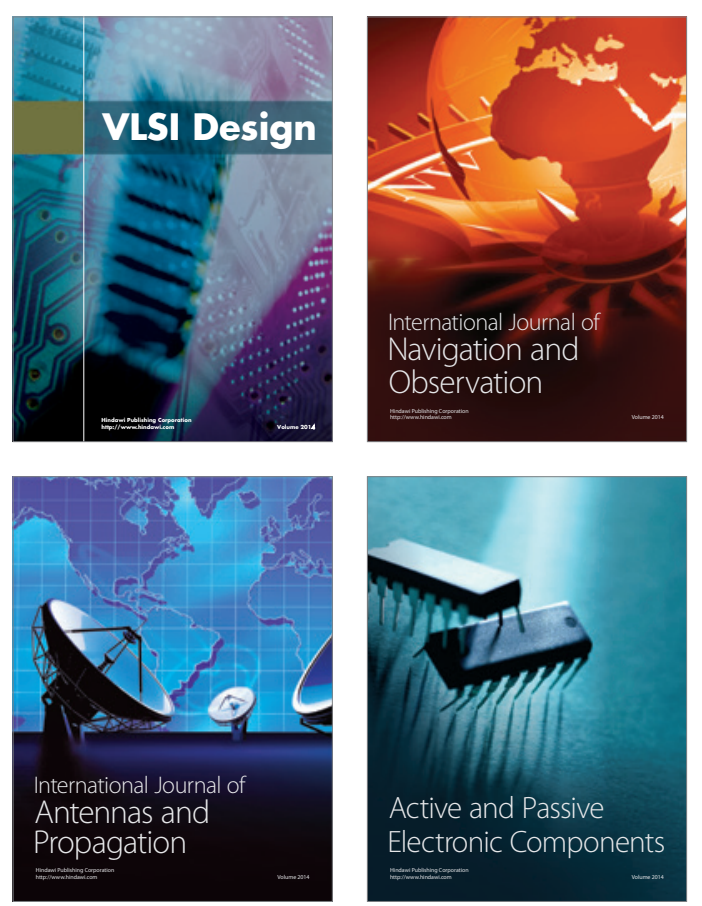
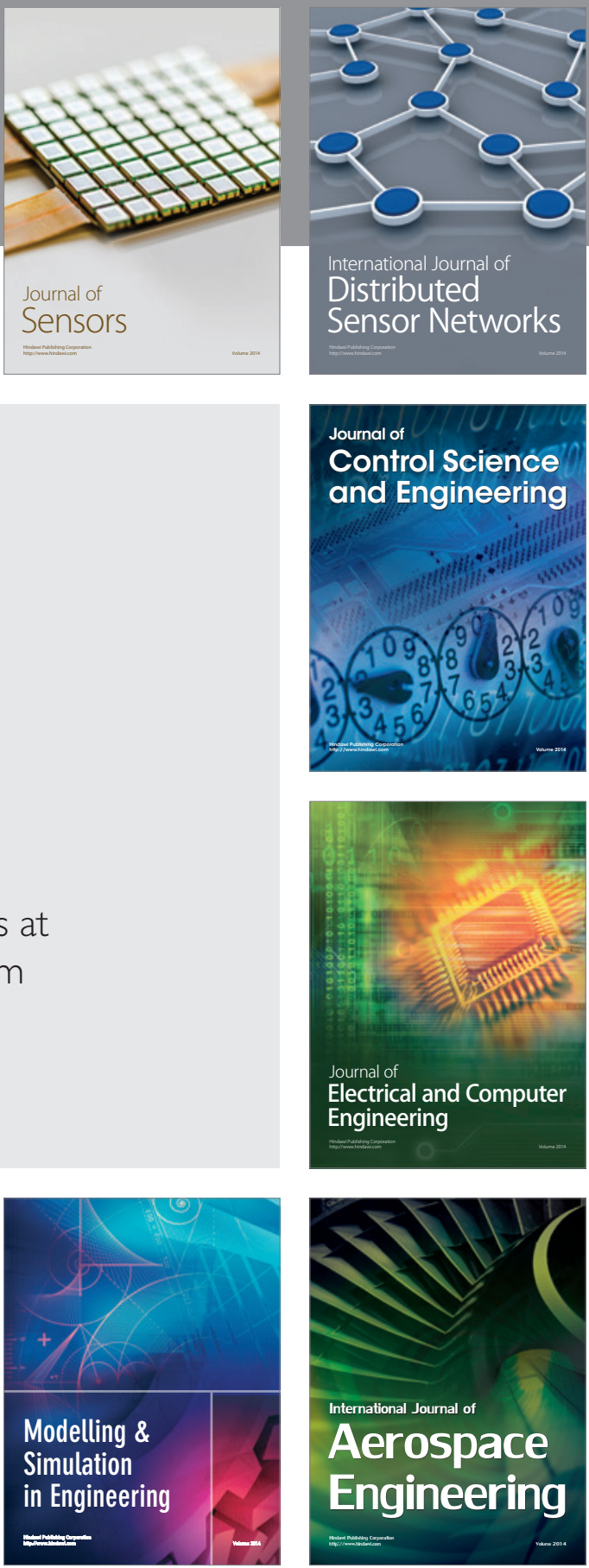

Journal of

Control Science

and Engineering
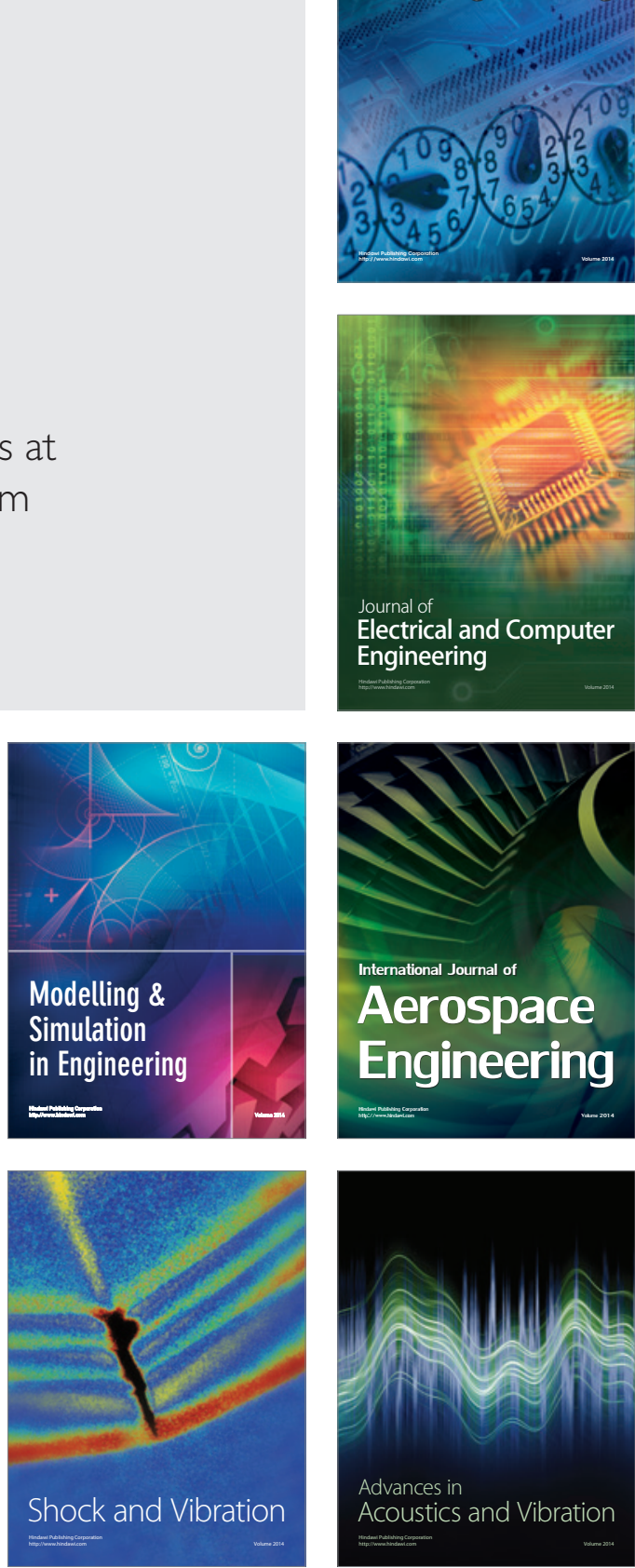